\title{
Electron Microscopy Study of Carbon Nanotubes Synthesized by Plasma-Enhanced Chemical Vapor Deposition
}

Jianfeng Wu, Lifeng Dong, Timothy Gutu, Jennifer Jones and Jun Jiao

Physics Department, Portland State University, P.O Box 751, Portland, OR 97207

Due to their unique electronic and structural properties, multi-walled carbon nanotubes (MWCNTs) have been extensively studied as candidate materials for cold-cathode electron sources [1]. Plasma-enhanced chemical vapor deposition (PECVD) is a promising method for growing well-aligned MWCNTs. We report here the use of electron microscopy techniques to study the morphology and internal structures of nanotubes synthesized by PECVD in comparison to those synthesized by a thermal CVD method.

In this experiment, nanotubes were synthesized on silicon substrates coated with a $15 \mathrm{~nm}$ TiN barrier layer. The TiN layer prevents the diffusion and reaction of $\mathrm{Ni}$ catalyst with the silicon substrate, which would result in reduced CNT yield. A Ni thin film with a thickness of $10 \mathrm{~nm}$ was prepared by a sputtering physical vapor deposition method. After the deposition of the catalyst thin film, the substrates were heated to $725^{\circ} \mathrm{C}$ and exposed to $50 \mathrm{sccm}$ of $\mathrm{C}_{2} \mathrm{H}_{2}$ and $200 \mathrm{sccm}$ of $\mathrm{NH}_{3}$ gases in a CVD or PECVD chamber for approximately $10 \mathrm{~min}$. During this process, the Ni thin film was first transformed into Ni nanoparticles and then served as a catalyst for the CNT growth. The introduction of $\mathrm{C}_{2} \mathrm{H}_{2}$ provides a carbon source, while the $\mathrm{NH}_{3}$ prevents the deposition of amorphous carbon. An FEI Sirion field emission SEM and a Tecnai F-20 field emission TEM/STEM equipped with an energy-dispersive x-ray (EDX) were used to characterize the morphology and internal structures of the synthesized nanotubes.

In comparison to the thermal CVD process, the significant difference in the PECVD method is that the silicon substrate was biased by a negative potential of $630 \mathrm{~V}$ to form glow discharge plasma. As demonstrated in Fig. 1, nanotubes synthesized by thermal CVD were curly and randomly oriented. The CNTs formed by PECVD were well aligned in a vertical configuration as shown in Fig. 2. This suggests that the electric field in the plasma caused nanotubes to grow along the electric field direction. Another difference is the shapes of the tube tips. The nanotubes made by thermal CVD terminated with an irregular shape while the tube tips made by PECVD have a more uniform shape as indicated in the insets of Fig. 1 and Fig. 2, respectively. High resolution TEM images of typical CNTs grown by PECVD are shown in Fig. 3 and Fig. 4. Note that the Ni catalyst was located at the tip of CNTs. The tip-growth mechanism is favored because of the plasma electrostatic force making the $\mathrm{Ni}$ nanoparticle detached from the TiN diffusion barrier [2]. The internal structures of the PECVD tubes display bamboo-like fringes characterized by periodic curving graphitic bands normal to the tube axis. Small Ni particles are also observed inside the nanotubes, as demonstrated by the STEM image in Fig. 5. The EDX line scan results in Fig. 6 clearly demonstrate the presence of a small $\mathrm{Ni}$ particle inside the main body of the nanotube and a large Ni particle within the nanotube tip region.

It is clear that PECVD provides a more desirable and effective method for the synthesis of vertically aligned carbon nanotubes. This morphology will enhance the field emission properties of carbon nanotubes. Further study is underway to synthesize nanotubes from different catalysts via the PECVD process and to investigate their field emission properties [3].

\section{References}

[1] Wei, L. et al, J. Vac. Sci. Technol. B 18 (2000) 2704.

[2] V.I.Merkulov, Appl. Phys. Lett. 79 (2001) 2970.

[3] Financial support for this research was provided in part by the NSF under awards No. ECS0348377, ECS-0217061 and DMR-0353738 

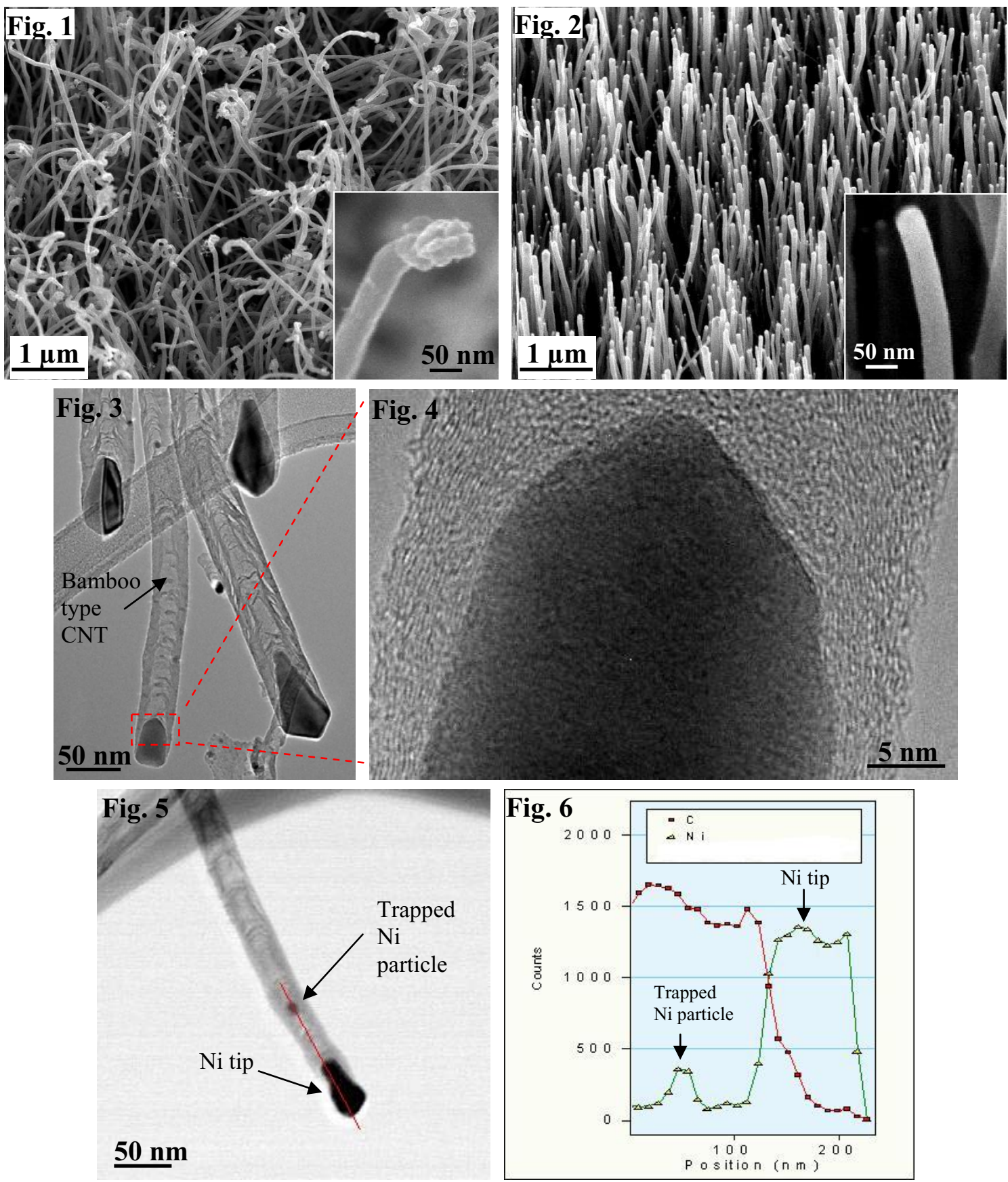

Fig.1 SEM image of curved MWCNTs synthesized by a thermal CVD process.

Fig.2 SEM image of straight MWCNTs synthesized by a PECVD process. The nanotubes were formed along the electrical field direction, which was vertical to the substrate surface.

Fig.3 TEM image of MWCNTs grown by the PECVD process. The Ni catalyst is located at the tip of each nanotube while bamboo-type fringes formed inside nanotubes.

Fig.4 An HRTEM image of the nanotube region labeled in Fig. 3 demonstrates that graphitic layers are mainly parallel to the tube axis.

Fig.5 STEM image of a nanotube with a Ni particle trapped inside.

Fig.6 An EDX line scan spectrum of the nanotube region labeled in Fig. 5 shows the distribution of carbon and nickel along the nanotube axis. 